\title{
Commonly applied positive end-expiratory pressures do not prevent functional residual capacity decline in the setting of intra-abdominal hypertension: a pig model
}

Adrian Regli ${ }^{*}$, Lisen E Hockings ${ }^{1}$, Gabrielle C Musk², Brigit Roberts ${ }^{1}$, Bill Noffsinger ${ }^{3}$, Bhajan Singh ${ }^{3}$, Peter $\vee$ van Heerden ${ }^{1}$

\begin{abstract}
Introduction: Intra-abdominal hypertension is common in critically ill patients and is associated with increased morbidity and mortality. The optimal ventilation strategy remains unclear in these patients. We examined the effect of positive end-expiratory pressures (PEEP) on functional residual capacity (FRC) and oxygen delivery in a pig model of intra-abdominal hypertension.

Methods: Thirteen adult pigs received standardised anaesthesia and ventilation. We randomised three levels of intra-abdominal pressure ( $3 \mathrm{mmHg}$ (baseline), $18 \mathrm{mmHg}$, and $26 \mathrm{mmHg}$ ) and four commonly applied levels of $\operatorname{PEEP}\left(5,8,12\right.$ and $\left.15 \mathrm{cmH}_{2} \mathrm{O}\right)$. Intra-abdominal pressures were generated by inflating an intra-abdominal balloon. We measured intra-abdominal (bladder) pressure, functional residual capacity, cardiac output, haemoglobin and oxygen saturation, and calculated oxygen delivery.

Results: Raised intra-abdominal pressure decreased FRC but did not change cardiac output. PEEP increased FRC at baseline intra-abdominal pressure. The decline in FRC with raised intra-abdominal pressure was partly reversed by PEEP at $18 \mathrm{mmHg}$ intra-abdominal pressure and not at all at $26 \mathrm{mmHg}$ intra-abdominal pressure. PEEP significantly decreased cardiac output and oxygen delivery at baseline and at $26 \mathrm{mmHg}$ intra-abdominal pressure but not at $18 \mathrm{mmHg}$ intra-abdominal pressure.

Conclusions: In a pig model of intra-abdominal hypertension, PEEP up to $15 \mathrm{cmH}_{2} \mathrm{O}$ did not prevent the FRC decline caused by intra-abdominal hypertension and was associated with reduced oxygen delivery as a consequence of reduced cardiac output. This implies that PEEP levels inferior to the corresponding intra-abdominal pressures cannot be recommended to prevent FRC decline in the setting of intra-abdominal hypertension.
\end{abstract}

\section{Introduction}

Intra-abdominal hypertension (IAH) is defined by the World Society of Abdominal Compartment Syndrome as a sustained increase in intra-abdominal pressure (IAP) above or equal to $12 \mathrm{mmHg}$ and abdominal compartment syndrome is defined as an IAP of more than $20 \mathrm{mmHg}$ plus a new organ dysfunction [1]. IAH and abdominal compartment syndrome are common in critically ill patients and are associated with a high rate of morbidity

\footnotetext{
* Correspondence: adrian.regli@gmail.com
'Intensive Care Unit, Sir Charles Gairdner Hospital, Hospital Avenue, Nedlands

* Correspondence: adrian.regli@gmail.com
'Intensive Care Unit, Sir Charles Gairdner Hospital, Hospital Avenue, Nedlands (Perth) WA 6009, Australia
}

(c) 2010 Regli et al.; licensee BioMed Central Ltd. This is an open access article distributed under the terms of the Creative Commons

and mortality [1-6]. IAH is associated with an increased systemic vascular resistance, a decreased venous return and a reduced cardiac output subsequently leading to reduced renal, hepatic and gastro-intestinal perfusion and thereby promoting multi organ failure [7-12].

Patients with IAH are susceptible to a significant impairment in lung function mainly caused by atelectasis resulting from a cephaled shift of the diaphragm, with subsequent decrease in lung volume leading to a decrease in arterial oxygenation [12-14]. Atelectasis is generally treated by recruitment manoeuvres followed by increasing positive end expiratory pressure (PEEP) in 
patients receiving mechanical ventilation [14-17]. However, in the setting of IAH, the role of PEEP remains unclear. On one hand increased levels of PEEP have been proposed to improve lung function $[13,18]$. On the other hand low levels of PEEP have been suggested to avoid haemodynamic compromise [7].

The correct diagnosis and treatment of the underlying condition and, where medical treatment fails and as a last resort, the performance of a decompressive laparotomy is recommended in patients with severe IAH $(>25$ $\mathrm{mmHg}$ ) [2]. However, in patients with less severe IAH or prior abdominal surgery in patients with severe IAH, the World Society of Abdominal Compartment Syndrome recommends that cardiac output $(\mathrm{CO})$ and oxygen delivery $\left(\mathrm{DO}_{2}\right)$ should be optimized, as this has been associated with a reduced morbidity and mortality in these patients $[2,8]$.

The aim of this project was to study the effect of commonly applied PEEP levels on FRC, arterial oxygen saturation, $\mathrm{CO}$ and $\mathrm{DO}_{2}$ in a healthy pig model of IAH. We hypothesized that PEEP would increase FRC and decrease $\mathrm{CO}$ and that there would be a PEEP level at which $\mathrm{DO}_{2}$ would be optimal. We also hypothesized that high levels of PEEP would increase IAP.

\section{Materials and methods}

The study conformed to the regulations of the Australian code of practice for the care and use of animals for scientific purposes and was approved by the Animal Ethics Committee of the University of Western Australia.

\section{Preparation of animals and anaesthesia}

We studied 13 pigs (Large White breed), which were fasted overnight, but with free access to water. Each of the animals was weighed and then sedated with an intramuscular injection of Zoletil ${ }^{\circ}$ (1:1 combination of tiletamine and zolazepam, Virbac, Milperra, NSW, Australia) $(4 \mathrm{mg} / \mathrm{kg})$ and xylazine $(2 \mathrm{mg} / \mathrm{kg})$. Venous access was then established and secured in an auricular vein. To facilitate endotracheal intubation, an intravenous (IV) bolus of propofol $(1 \mathrm{mg} / \mathrm{kg})$ was administered. The trachea was intubated via the oral route with a cuffed endotracheal tube (size $8.0 \mathrm{~mm}$, Hi-Lo, Mallinckrodt, Athlone, Ireland). Anaesthesia was maintained with a combination of propofol ( 9 to $36 \mathrm{mg} / \mathrm{kg} / \mathrm{h}$ IV), morphine $(0.1$ to $0.2 \mathrm{mg} / \mathrm{kg} / \mathrm{h} \mathrm{IV})$ and ketamine (0.3 to 0.6 $\mathrm{mg} / \mathrm{kg} / \mathrm{h} \mathrm{IV)} \mathrm{according} \mathrm{to} \mathrm{clinical} \mathrm{requirements.} \mathrm{Neuro-}$ muscular blocking agents were not administered. A core temperature of $36^{\circ} \mathrm{C}$ to $38^{\circ} \mathrm{C}$ was maintained by the application of heating mats.

Succinylated gelatin (Gelofusine, Braun, Oss, The Netherlands) was given pre-emptively for haemodynamic stabilization $(500 \mathrm{~mL}$ over the first 30 minutes followed by 1
$\mathrm{mL} / \mathrm{kg} / \mathrm{h}$ ). At the end of the protocol the pigs were euthanized with pentobarbitone $(100 \mathrm{mg} / \mathrm{kg}$ body weight), injected IV.

\section{Ventilation}

A critical care ventilator (Servo 900, Siemens, Berlin, Germany) was used with the following ventilator settings: $\mathrm{FiO}_{2}$ 0.4, volume control mode, I:E ratio of 1:2, tidal volume of $8 \mathrm{ml} / \mathrm{kg}$ with the respiratory rate adjusted to maintain an end tidal $\mathrm{CO}_{2}$ of 35 to 45 $\mathrm{mmHg}$. The initial PEEP setting was $5 \mathrm{cmH}_{2} \mathrm{O}(3.7$ $\mathrm{mmHg}$ ) and altered according to the experimental protocol. Peak airway pressure (pPaw), mean airway pressure (mPaw), and dynamic compliance (Cdyn) were measured by the ventilator.

\section{Surgical procedure}

Throughout the study the animals remained supine. Following a chlorhexidine based antiseptic skin preparation the pigs were instrumented as follows:

\section{Haemodynamic monitoring}

A 16-gauge single lumen catheter (ES-04301, Arrow International, Reading, PA, USA) was inserted into the femoral artery to measure the mean arterial blood pressure (MAP). An $8.5 \mathrm{~F}$ percutaneous introducer (SI09806, Arrow International) was inserted into the internal jugular vein to allow the placement of a pulmonary artery catheter (AH-05050, Arrow International) under continuous pressure wave monitoring into the pulmonary artery in order to measure $\mathrm{CO}$.

\section{Intra-abdominal pressure measurement and generation}

For the measurement of IAP, a caudal midline laparotomy was performed to place a $12 \mathrm{~F}$ Foley catheter (226512, Bard, Covington, GA, USA) in the urinary bladder.

For the generation of different levels of IAP, we performed another cephaled midline laparotomy in order to place a latex balloon (200 g weather balloon, Scientific Sales, Lawrenceville, NJ, USA) in the peritoneal cavity. The abdomen was tightly closed with sutures. Inflation of the intra-abdominal balloon with air allowed the generation of different levels of IAP [19].

We measured IAP using urinary bladder pressure as defined by the World Society of Abdominal Compartment Syndrome with the only difference that we measured mean IAP instead of end-expiratory IAP [1]. We used a standardised injection volume of normal saline (25 ml syringe with auto-valve, AbViser, Wolfe Tory Medical, Salt Lake City, UT, USA). We measured urinary bladder pressure before and after alterations of PEEP.

\section{Experimental protocol}

After a set of baseline measurements, the abdominal balloon was either not inflated (baseline IAP) or inflated 
with air to produce grade II $(18+/-2 \mathrm{mmHg})$ or grade IV $(26+/-2 \mathrm{mmHg})$ IAH in predefined random order [1]. PEEP was then applied in a predefined random manner at $5,8,12$ or $15 \mathrm{cmH}_{2} \mathrm{O}(3.7,5.9,8.8$, and 11.0 $\mathrm{mmHg}$, respectively) at each level of IAP; these are commonly used levels of PEEP in critically ill patients. For randomisation, we used a spit plot design ensuring all 12 combinations of IAH and PEEP levels were applied to all animals [20].

For each IAP and PEEP setting, we performed a standardised lung recruitment manoeuvre as follows [21]. PEEP was increased every respiratory cycle by increments of $2 \mathrm{cmH}_{2} \mathrm{O}(1.5 \mathrm{mmHg})$ in order to achieve either a PEEP value of $15 \mathrm{cmH}_{2} \mathrm{O}(11.0 \mathrm{mmHg})$ or a maximum peak airway pressure of $40 \mathrm{cmH}_{2} \mathrm{O}$ $(29.4 \mathrm{mmHg})$ and then continued for 10 consecutive breaths. Thereafter the PEEP was decreased by 2 $\mathrm{cmH}_{2} \mathrm{O}(1.5 \mathrm{mmHg})$ decrements per respiratory cycle until the target PEEP setting was achieved according to the experimental protocol. All respiratory and haemodynamic measurements were then performed after a fiveminute period allowing for abdominal, respiratory and haemodynamic stabilization.

\section{Measurements and calculations \\ Haemodynamic parameters}

All pressures including IAP were measured with a transducer (Hospira, Lake Forest, IL, USA) and monitored with a critical care monitor (Sirecust 126; Siemens Medical Electronics, Danvers, MA, USA). MAP, central venous pressure (CVP) and heart rate (from electro-cardiogram) were measured. All pressures were zeroed at the mid axillary line, including urinary bladder pressure [1]. CO was measured by thermodilution using a standardised $10 \mathrm{ml}$ bolus of ice cold normal saline (Sirecust 126; Siemens Medical Electronics). For each IAP and PEEP setting, three $\mathrm{CO}$ measurements were performed and averaged.

\section{Functional residual capacity}

FRC was measured using the multiple breath nitrogen wash-out method [22]. After switching $\mathrm{FiO}_{2}$ from 0.4 to 1.0 an air tight bag collected the expiratory gas from the ventilator until $<0.5 \%$ nitrogen was detectable. The total expired gas volume was measured using a digital pneumotachograph (HP 47303A, Hewlett-Packard, Paranus, NJ, USA) and nitrogen concentration was measured with a nitrogen analyzer (HP 47302A, Hewlett-Packard) after mixing the expired gas. Three FRC measurements for each IAP and PEEP setting were performed and averaged.

\section{Oxygenation}

Arterial oxygen tension $\left(\mathrm{PaO}_{2}\right)$ and haemoglobin concentration $(\mathrm{Hb})$ were measured with a blood gas machine (ABL77, Radiometer, Copenhagen, Denmark) immediately following collection. Blood was drawn from the femoral artery and pulmonary artery in order to measure arterial, and mixed venous oxygen tensions, respectively.

\section{Calculations}

The following calculations were made from the measured variables: Abdominal perfusion pressure $(\mathrm{APP})=\mathrm{MAP}-$ IAP [1]. Systemic vascular resistance $(\mathrm{SVR})=(\mathrm{MAP}-$ $\mathrm{CVP}) / \mathrm{CO} \times 79.9$ dyn $\times \mathrm{s} / \mathrm{cm}^{5} . \mathrm{PaO}_{2}$ was corrected for $\mathrm{pH}\left(\mathrm{PaO}_{2}\right.$ cor $)=\mathrm{PaO}_{2} \times 10(0.30 \times(\mathrm{pH}-7.4)$ [23]. Oxygen saturation $=100 \times\left(0.13534 \times \mathrm{PaO}_{2} \text { cor }\right)^{3.02} /((0.13534 \times$ $\mathrm{PaO}_{2}$ cor $\left.\left.)^{3.02}+91.2\right)\right)[23]$. Oxygen content $=$ oxygen saturation $\times(\% / 100) \times \mathrm{Hb}(\mathrm{g} / \mathrm{dl}) \times 1.39(\mathrm{ml} / \mathrm{g})+0.003$ $(\mathrm{ml} / \mathrm{dl}) \times \mathrm{PO}_{2}$ cor $)$ [24]. $\mathrm{DO}_{2}=\mathrm{CO} \times$ arterial oxygen content [24]. FRC $=(($ total expired gas volume $\times$ nitrogen concentration $) /(100 \times 0.6))-1.92($ measured dead space of ventilator).

\section{Statistics}

To detect a difference in $\mathrm{DO}_{2}$ of $3.0 \mathrm{ml} / \mathrm{kg} / \mathrm{min}$ (assuming a mean (SD) $\mathrm{DO}_{2}$ of $18.0(4.0) \mathrm{ml} / \mathrm{kg} /$ minute) [25] between two different PEEP values $(\alpha=0.05$, power $=$ $80 \%$ ) we calculated a sample size of 13 pigs. Data are reported as mean (SD), as the data proved to be normally distributed, when analyzed by the KolmogorovSmirnov test. To compare the data between the different combinations of PEEP and IAP, an ANOVA for repeated measures was performed and a post hoc Student-Newman-Keuls-test to adjust for multiple comparisons. A probability of $<0.05$ was considered statistically significant.

\section{Results}

Mean (SD) animal weight was $42(8) \mathrm{kg}$. Haemoglobin concentration was 103 (8) g/L. After inflation of the intra-abdominal balloon to the target IAP, the IAP remained constant over the five-minute stabilising period. The resulting level of IAP at the time of measurement was: 3 (2), 18 (3), and 26 (4) $\mathrm{mmHg}$ for baseline, grade II IAH, and grade IV IAH settings, respectively. There were no differences between the measured parameters at baseline IAP and $5 \mathrm{cmH}_{2} \mathrm{O}$ PEEP taken before and during the randomized protocol. An adjustment of the values according to the weight of the individual animal did not alter the findings, therefore absolute values are given. The influence of IAP and PEEP on haemodynamic and respiratory parameters is shown in Tables 1 , 2 and 3, and Figures 1, 2, 3 and 4. Increasing PEEP from 5 to $15 \mathrm{cmH}_{2} \mathrm{O}$ (3.7 to $11.0 \mathrm{mmHg}$ ) did not significantly increase IAP $(+0.4(0.8) \mathrm{mmHg})$.

\section{Effect of IAP on FRC, and $\mathrm{PaO}_{2}$}

$\mathrm{SaO}_{2}$ was $99.7(0.2) \%$ at all levels of IAP and PEEP. IAH was associated with lower levels of $\mathrm{PaO}_{2}$. However, differences in $\mathrm{PaO}_{2}$ were only significant at 8 and 
Table 1 Influence of positive end-expiratory pressure on respiratory and haemodynamic data at baseline intraabdominal pressure

\begin{tabular}{|c|c|c|c|c|c|c|c|}
\hline PEEP, $\mathrm{cmH}_{2} \mathrm{O}$ & 5 & 8 & 5 vs 8 & 12 & 5 vs 12 & 15 & 5 vs 15 \\
\hline FRC, L & $1.4(0.4)$ & $1.5(0.5)$ & NS & $1.7(0.5)$ & 0.002 & $1.7(0.6)$ & $<0.001$ \\
\hline $\mathrm{PaO}_{2}, \mathrm{mmHg}$ & $237(14)$ & 240 (19) & NS & $236(16)$ & NS & $227(25)$ & $<0.05$ \\
\hline pPaw, $\mathrm{cmH}_{2} \mathrm{O}$ & $21(6)$ & $24(5)$ & $<0.001$ & $27(5)$ & $<0.001$ & $32(5)$ & $<0.001$ \\
\hline mPaw, $\mathrm{cmH}_{2} \mathrm{O}$ & $10(1)$ & $13(2)$ & $<0.001$ & $16(2)$ & $<0.001$ & $19(1)$ & $<0.001$ \\
\hline $\mathrm{C}$ dyn, $\mathrm{ml} / \mathrm{cmH}_{2} \mathrm{O}$ & $25(8)$ & $25(9)$ & NS & $24(9)$ & NS & $21(6)$ & $<0.001$ \\
\hline $\mathrm{CO}, \mathrm{L} / \mathrm{min}$ & $3.5(1.0)$ & $3.2(1.0)$ & NS & $2.7(0.7)$ & 0.009 & $2.5(0.7)$ & 0.002 \\
\hline $\mathrm{DO}_{2}, \mathrm{ml} / \mathrm{min}$ & $498(156)$ & 459 (156) & NS & 381 (112) & 0.006 & $349(100)$ & $<0.001$ \\
\hline $\mathrm{SvO}_{2}, \%$ & $62(7)$ & $55(11)$ & $<0.05$ & $47(13)$ & $<0.05$ & $44(17)$ & $<0.05$ \\
\hline $\mathrm{VO}_{2}, \mathrm{ml} / \mathrm{min}$ & $191(47)$ & $209(70)$ & NS & $205(81)$ & NS & $196(53)$ & NS \\
\hline MAP, mmHg & $71(19)$ & $67(15)$ & NS & $60(13)$ & 0.025 & $56(21)$ & 0.004 \\
\hline APP, mmHg & 69 (19) & $64(15)$ & NS & $56(13)$ & 0.01 & $53(21)$ & 0.002 \\
\hline CVP, mmHg & $8(4)$ & $8(3)$ & NS & $9(2)$ & NS & $10(3)$ & NS \\
\hline $\mathrm{PAOP}, \mathrm{mmHg}$ & $6(2)$ & $6(2)$ & NS & $8(2)$ & 0.004 & $9(1)$ & $<0.001$ \\
\hline $\mathrm{HR}$, beats/min & 79 (13) & $81(18)$ & NS & $85(20)$ & NS & $89(24)$ & 0.026 \\
\hline$S V R$, dyn $* s / \mathrm{cm}^{5}$ & 1,389 (408) & $1,404(352)$ & NS & 1,445 (373) & NS & $1,337(321)$ & NS \\
\hline $\mathrm{SV}, \mathrm{ml}$ & $50(21)$ & $44(13)$ & NS & 37 (12) & 0.002 & $33(10)$ & $<0.001$ \\
\hline
\end{tabular}

APP, abdominal perfusion pressure; $C$ dyn, dynamic compliance; $C O$, cardiac output; $C V P$, central venous pressure; $\mathrm{DO}_{2}$, oxygen delivery; FRC, functional residual capacity; $\mathrm{HR}$, heart rate; MAP, mean arterial pressure; $\mathrm{mPaw}$, mean airway pressure; $\mathrm{PaO}_{2}$, arterial oxygen tension; PAOP, pulmonary artery occlusion pressure; PEEP, positive end-expiratory pressure; pPaw, peak airway pressure; SV, stroke volume; $\mathrm{SvO}_{2}$, mixed venous oxygen saturation; SVR, systemic vascular resistance; $\mathrm{VO}_{2}$, oxygen consumption. Mean (SD) are given. ANOVA and post hoc Student-Newman-Keuls were used for statistical testing. NS, not significant $(P>0.05)$.

Table 2 Influence of positive end-expiratory pressure on respiratory and haemodynamic data at $18 \mathrm{mmHg}$ intraabdominal pressure

\begin{tabular}{|c|c|c|c|c|c|c|c|}
\hline PEEP, $\mathrm{cmH}_{2} \mathrm{O}$ & 5 & 8 & 5 vs 8 & 12 & 5 vs 12 & 15 & 5 vs 15 \\
\hline FRC, L & $0.9(0.3) *$ & $1.0(0.3) *$ & 0.034 & $1.0(0.3) *$ & 0.049 & $1.1(0.3) *$ & $<0.001$ \\
\hline $\mathrm{PaO}_{2}, \mathrm{mmHg}$ & $215(32)$ & $218(20) *$ & NS & $222(23) *$ & NS & $216(26)$ & NS \\
\hline pPaw, $\mathrm{cmH}_{2} \mathrm{O}$ & $29(5) *$ & $31(5) *$ & $<0.001$ & $34(5) *$ & $<0.001$ & $37(5) *$ & $<0.001$ \\
\hline $\mathrm{mPaw}, \mathrm{cmH}_{2} \mathrm{O}$ & $12(3) *$ & $15(3) *$ & $<0.001$ & $18(3) *$ & $<0.001$ & $21(4) *$ & $<0.001$ \\
\hline $\mathrm{C}$ dyn, $\mathrm{ml} / \mathrm{cmH}_{2} \mathrm{O}$ & $15(4) *$ & $15(3) *$ & NS & $16(4) *$ & NS & $16(4) *$ & NS \\
\hline $\mathrm{CO}, \mathrm{L} / \mathrm{min}$ & $3.5(0.9)$ & $3.4(0.7)$ & NS & $3.4(0.9)$ & NS & $3.1(0.8) *$ & NS \\
\hline $\mathrm{DO}_{2}, \mathrm{ml} / \mathrm{min}$ & $490(130)$ & $472(91)$ & NS & $472(124) *$ & NS & $428(116) *$ & NS \\
\hline $\mathrm{SvO}_{2}, \%$ & $61(9)$ & $61(10)$ & NS & $58(11) *$ & NS & $56(14) *$ & NS \\
\hline $\mathrm{VO}_{2}, \mathrm{ml} / \mathrm{min}$ & $195(64)$ & $192(50)$ & NS & $202(55)$ & NS & $186(47)$ & NS \\
\hline MAP, $\mathrm{mmHg}$ & $83(12)$ & $79(11) *$ & NS & $81(16) *$ & NS & $72(13) *$ & $<0.001$ \\
\hline APP, $\mathrm{mmHg}$ & $65(13)$ & $62(12)$ & NS & $63(18)$ & NS & $56(12)$ & 0.013 \\
\hline CVP, mmHg & $10(3) *$ & $11(2) *$ & NS & $13(4) *$ & $<0.001$ & $15(1) *$ & $<0.001$ \\
\hline $\mathrm{PAOP}, \mathrm{mmHg}$ & $7(2)$ & $9(1) *$ & $<0.001$ & $11(2) *$ & $<0.001$ & $12(1) *$ & $<0.001$ \\
\hline HR, beats/min & $73(16)$ & $72(14)$ & NS & $74(14)$ & NS & $76(14)$ & NS \\
\hline SVR, dyn $* s / \mathrm{cm}^{5}$ & $1,643(364)$ & $1,600(217) *$ & NS & $1,580(248)$ & NS & $1,491(275)$ & NS \\
\hline $\mathrm{SV}, \mathrm{ml}$ & $52(23)$ & $49(10) *$ & NS & $47(14) *$ & NS & $42(12) *$ & NS \\
\hline
\end{tabular}

APP, abdominal perfusion pressure; Cdyn, dynamic compliance; $\mathrm{CO}$, cardiac output; CVP, central venous pressure; $\mathrm{DO}_{2}$, oxygen delivery; $\mathrm{FRC}$, functional residual capacity; $\mathrm{HR}$, heart rate; MAP, mean arterial pressure; $\mathrm{mPaw}$, mean airway pressure; $\mathrm{PaO}_{2}$, arterial oxygen tension; PAOP, pulmonary artery occlusion pressure; PEEP, positive end-expiratory pressure; pPaw, peak airway pressure; SV, stroke volume; $\mathrm{SvO}_{2}$, mixed venous oxygen saturation; SVR, systemic vascular resistance; $\mathrm{VO}_{2}$, oxygen consumption. Mean (SD) are given. ANOVA and post hoc Student-Newman-Keuls were used for statistical testing. ${ }^{*}$, significant $(P<0.05)$ difference compared with baseline IAP. NS, not significant.

$12 \mathrm{cmH}_{2} \mathrm{O}$ of PEEP when comparing the differences between baseline IAP and 18 and $26 \mathrm{mmHg}$ IAP (Tables 1, 2 and 3). Increasing levels of IAP were associated with a decrease in FRC by $33(15) \%$ and 30 (18)\% for grade II and grade IV IAH, respectively (Figure 1).
Effect of PEEP at different levels of IAP on FRC, and $\mathrm{PaO}_{2}$ PEEP did not improve $\mathrm{PaO}_{2}$ (Tables 1, 2 and 3). The effect of PEEP on FRC varied at different levels of IAP. At baseline IAP, PEEP increased FRC (Figure 1). At grade II IAH, but not at grade IV IAH, the IAP induced FRC decline partially reversed with increasing levels of 
Table 3 Influence of positive end-expiratory pressure on respiratory and haemodynamic data at $26 \mathrm{mmHg}$ intraabdominal pressure

\begin{tabular}{|c|c|c|c|c|c|c|c|}
\hline PEEP, $\mathrm{cmH}_{2} \mathrm{O}$ & 5 & 8 & 5 vs 8 & 12 & 5 vs 12 & 15 & 5 vs 15 \\
\hline FRC, L & $1.0(0.2) *$ & $1.0(0.3) *$ & NS & $1.0(0.3) *$ & NS & $1.0(0.2) *$ & NS \\
\hline $\mathrm{PaO}_{2}, \mathrm{mmHg}$ & $213(24)$ & $215(21) *$ & NS & $212(21) *$ & NS & $212(23)$ & NS \\
\hline pPaw, $\mathrm{cmH}_{2} \mathrm{O}$ & $33(4) *$ & $36(4) *$ & $<0.001$ & $38(5) *$ & $<0.001$ & $42(4) *$ & $<0.001$ \\
\hline mPaw, $\mathrm{cmH}_{2} \mathrm{O}$ & $13(4) *$ & $16(4) *$ & $<0.001$ & $19(4) *$ & $<0.001$ & $22(4) *$ & $<0.001$ \\
\hline $\mathrm{C}$ dyn, $\mathrm{ml} / \mathrm{cmH}_{2} \mathrm{O}$ & $13(3)$ * & $13(3) *$ & NS & $13(4) *$ & NS & $12(3) *$ & NS \\
\hline $\mathrm{CO}, \mathrm{L} / \mathrm{min}$ & $3.2(1.0)$ & $2.6(0.6) *$ & $<0.001$ & $2.7(0.9)$ & $<0.001$ & $2.5(0.8)$ & $<0.001$ \\
\hline $\mathrm{DO}_{2}, \mathrm{ml} / \mathrm{min}$ & 449 (161) & $367(93) *$ & 0.035 & 377 (140) & 0.029 & 349 (124) & 0.005 \\
\hline $\mathrm{SvO}_{2}, \%$ & $58(9)$ & $54(14)$ & 0.007 & $53(15) *$ & 0.02 & $52(13)$ & 0.045 \\
\hline $\mathrm{VO}_{2}, \mathrm{ml} / \mathrm{min}$ & $188(38)$ & $179(42)$ & NS & $183(54)$ & NS & $165(32)$ & NS \\
\hline MAP, mmHg & 78 (13) & 74 (18) & NS & $74(15) *$ & NS & $76(17) *$ & NS \\
\hline APP, mmHg & $52(14) *$ & $48(15) *$ & NS & $48(15)$ & NS & $49(17)$ & NS \\
\hline CVP, mmHg & $11(3) *$ & $12(2) *$ & NS & $13(2) *$ & 0.012 & $17(3) *$ & $<0.001$ \\
\hline PAOP, mmHg & $9(2)$ & $11(4) *$ & 0.024 & $12(2) *$ & 0.007 & $14(3) *$ & $<0.001$ \\
\hline $\mathrm{HR}$, beats/min & $72(10)$ & 78 (14) & NS & $76(13)$ & NS & $80(16)$ & NS \\
\hline$S V R$, dyn $* s / \mathrm{cm}^{5}$ & $1,771(446)$ & $1813(404) *$ & NS & $1,861(490) *$ & NS & $1,891(419) *$ & NS \\
\hline SV, ml & $44(12)$ & $37(14) *$ & 0.024 & $39(18)$ & 0.037 & $33(12)$ & 0.002 \\
\hline
\end{tabular}

APP, abdominal perfusion pressure; Cdyn, dynamic compliance; $\mathrm{CO}$, cardiac output; CVP, central venous pressure; $\mathrm{DO}_{2}$, oxygen delivery; $\mathrm{FRC}$, functional residual capacity; $\mathrm{HR}$, heart rate; MAP, mean arterial pressure; $\mathrm{mPaw}$, mean airway pressure; $\mathrm{PaO}_{2}$, arterial oxygen tension; PAOP, pulmonary artery occlusion pressure; PEEP, positive end-expiratory pressure; $\mathrm{pPaw}$, peak airway pressure; SV, stroke volume; $\mathrm{SvO}_{2}$, mixed venous oxygen saturation; SVR, systemic vascular resistance; $\mathrm{VO}_{2}$, oxygen consumption. Mean (SD) are given. ANOVA and post hoc Student-Newman-Keuls were used for statistical testing. ${ }^{*}$, significant $(P<0.05)$ difference compared with baseline IAP. NS, not significant.

PEEP. When PEEP was increased from 5 to $15 \mathrm{cmH}_{2} \mathrm{O}$ (3.7 to $11.0 \mathrm{mmHg}$ ), FRC increased by $0.3(0.3) \mathrm{L} \mathrm{(23}$ (18)\%) at baseline IAP and $0.2(0.1) \mathrm{L}((20)(11) \%)$ at IAP $18 \mathrm{cmH}_{2} \mathrm{O}$.

\section{Effect of IAP on $\mathrm{CO}, \mathrm{DO}_{2}, \mathrm{SvO}_{2}$, and SVR}

IAH did not significantly change $\mathrm{CO}$ and $\mathrm{DO}_{2}$ at 5 $\mathrm{cmH}_{2} \mathrm{O}$ of PEEP (Figures 2 and 3, and Tables 1, 2 and 3).

\section{Effect of PEEP at different levels of IAP on $\mathrm{CO}, \mathrm{DO}_{2} \mathrm{SvO}_{2}$, and SVR}

PEEP was associated with a dose-related decrease in $\mathrm{CO}$ and $\mathrm{DO}_{2}$ at baseline IAP and at grade IV IAH, but not at grade II IAH (Figures 2 and 3 ). When PEEP was increased from 5 to $15 \mathrm{cmH}_{2} \mathrm{O}$ (3.7 to $11.0 \mathrm{mmHg}$ ), $\mathrm{DO}_{2}$ decreased by 151 (158) $\mathrm{ml} /$ minute (25 (28)\%) at baseline IAP and by $100(72) \mathrm{ml} /$ minute (20 (20)\%) at grade IV IAH.

The changes in $\mathrm{SvO}_{2}$ caused by IAH and PEEP paralleled those of CO. SVR increased significantly with rising IAP, but not with increasing PEEP.

\section{Discussion}

There are many studies examining the influence of IAH on haemodynamic or on respiratory parameters. However, there are only a few studies investigating the effect of IAP and PEEP on cardio-respiratory parameters [26-28]. To our knowledge, this is the first study to assess the effect of different levels of PEEP in the setting of different levels of IAP on lung volumes assessed by FRC and CO parameters in a healthy pig model.

\section{Effect of IAP and PEEP on FRC, and $\mathrm{PaO}_{2}$}

We found that increasing IAP from baseline to grade II IAH decreased FRC and $\mathrm{PaO}_{2}$ levels by approximately $30 \%$ and $10 \%$, respectively. There was no further decrease in FRC and $\mathrm{PaO}_{2}$ when IAP was increased from grade II to grade IV IAH. This suggests either a high impedance to further lengthening and cephalic motion of the diaphragm or compensatory lung expansion due to expansion of the rib cage.

Even in the absence of IAH, a healthy patient requiring mechanical ventilation will experience some degree of FRC reduction due to atelectasis [24]. Although the role of PEEP in acute lung injury and acute respiratory distress syndrome remains controversial, recruitment manoeuvres and high levels of PEEP have been shown to re-open collapsed alveoli and keep the alveoli open $[17,24]$. As expected in this healthy pig lung model, in the absence of IAH, PEEP increased FRC but did not increase the already high $\mathrm{PaO}_{2}$ levels.

In the presence of IAH, PEEP up to $15 \mathrm{cmH}_{2} \mathrm{O}$ only partially reversed the IAP, induced FRC decline in grade II IAH, and did not increase FRC in grade IV IAH. PEEP did not increase $\mathrm{PaO}_{2}$ values in IAH.

The minimal $\mathrm{PaO}_{2}$ decrease as compared to the relatively larger FRC decrease in the setting of raised IAP can be explained by the FRC not dropping below the 


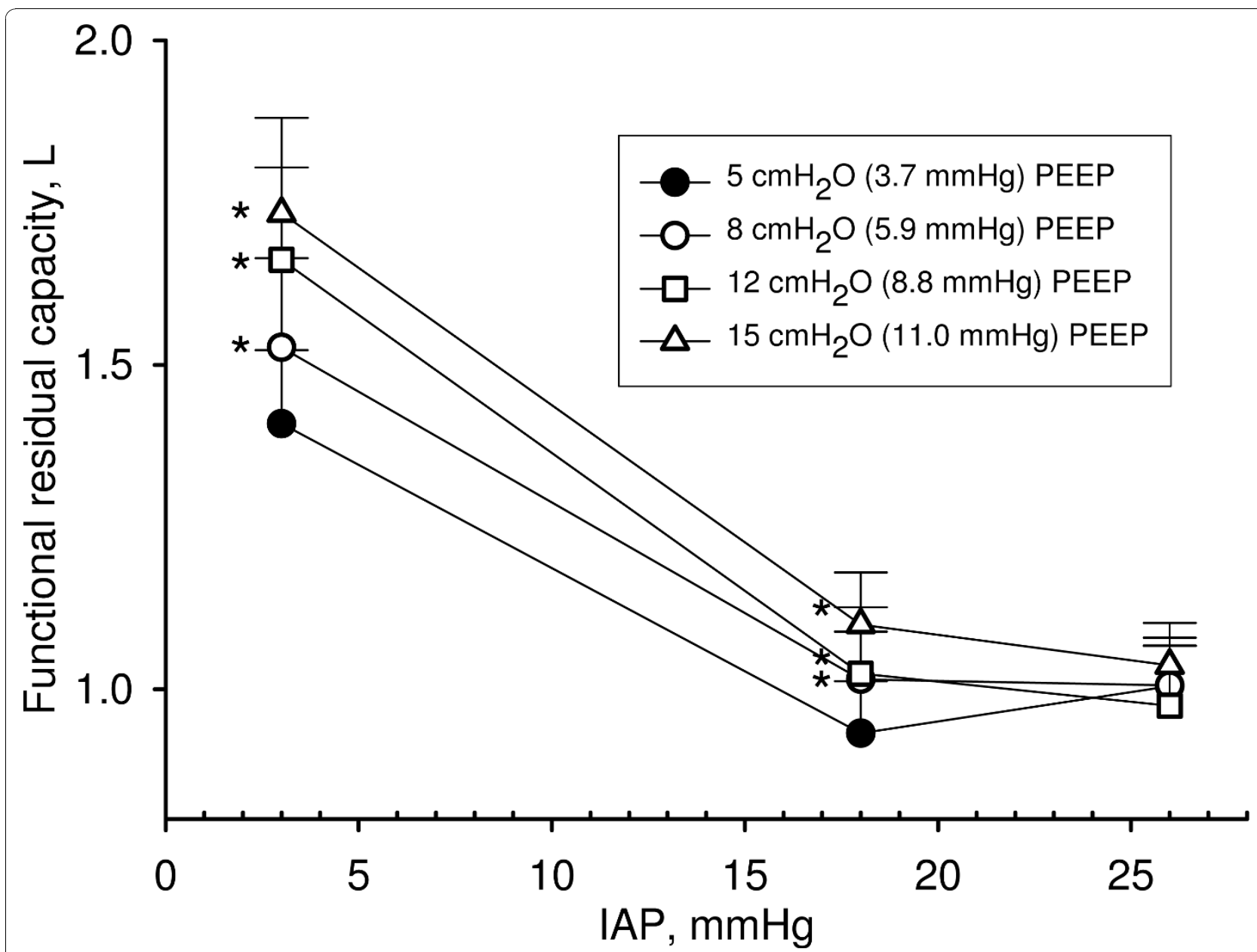

Figure 1 Influence of intra-abdominal pressure and positive end-expiratory pressure on functional residual capacity. Functional residual capacity (FRC) in litres (L) in function of different levels of intra-abdominal pressures (IAP) (3 mmHg (baseline), $18 \mathrm{mmHg}$ (grade II intraabdominal hypertension), and $26 \mathrm{mmHg}$ (grade IV intra-abdominal hypertension)) at different levels of positive end-expiratory pressures (PEEP). Mean and SE are shown. ANOVA and post hoc Student-Newman-Keuls were used for statistical testing. ${ }^{*}, P<0.05$ within an IAP setting vs. the corresponding value at $5 \mathrm{cmH}_{2} \mathrm{O}$ PEEP. For clarification additional symbol is added where necessary. At each PEEP setting, all FRC values were significantly different compared to the corresponding value at baseline IAP $(P<0.05)$.

closing capacity of healthy lungs and therefore not resulting in atelectasis, shunting and consecutively impaired gas exchange [24,29]. In the setting of acute respiratory distress syndrome where the closing capacity is increased, small decreases in FRC reductions may cause marked reductions in $\mathrm{PaO}_{2}$. However, this would need to be confirmed in further studies.

We chose PEEP levels of 5 to $15 \mathrm{cmH}_{2} \mathrm{O}$ as these represent PEEP levels frequently applied in critical ill patients. The minimal effect of PEEP on reversing the IAH induced FRC reduction can be explained by the reduced estimated trans-pulmonary end-expiratory pressures (PEEP - IAP) which would have approximated 8, -7 and $-15 \mathrm{mmHg}$ at PEEP of $15 \mathrm{cmH}_{2} \mathrm{O}(11.0 \mathrm{mmHg})$ and at IAP of $3 \mathrm{mmHg}$ (baseline), $18 \mathrm{mmHg}$ (grade II
IAH), and $26 \mathrm{mmHg}$ (grade IV IAH), respectively. Therefore, with regards to improving FRC and $\mathrm{PaO}_{2}$, PEEP values that are equal or higher than the corresponding IAP value might be necessary to protect against IAH induced FRC and $\mathrm{PaO}_{2}$ decrease as has previously been suggested [13]. However, when higher PEEP levels are applied in the setting of IAH, the potential detrimental effect of high PEEP levels on $\mathrm{CO}$ and $\mathrm{DO}_{2}$ should be considered and balanced against the lowest applicable PEEP in order to avoid haemodynamic compromise in this setting [7].

\section{Effect of IAP and PEEP on CO, $\mathrm{DO}_{2}$, and $\mathrm{SvO}_{2}$}

In agreement with other studies [29,30], we found that PEEP caused a dose-dependent decrease in stroke 


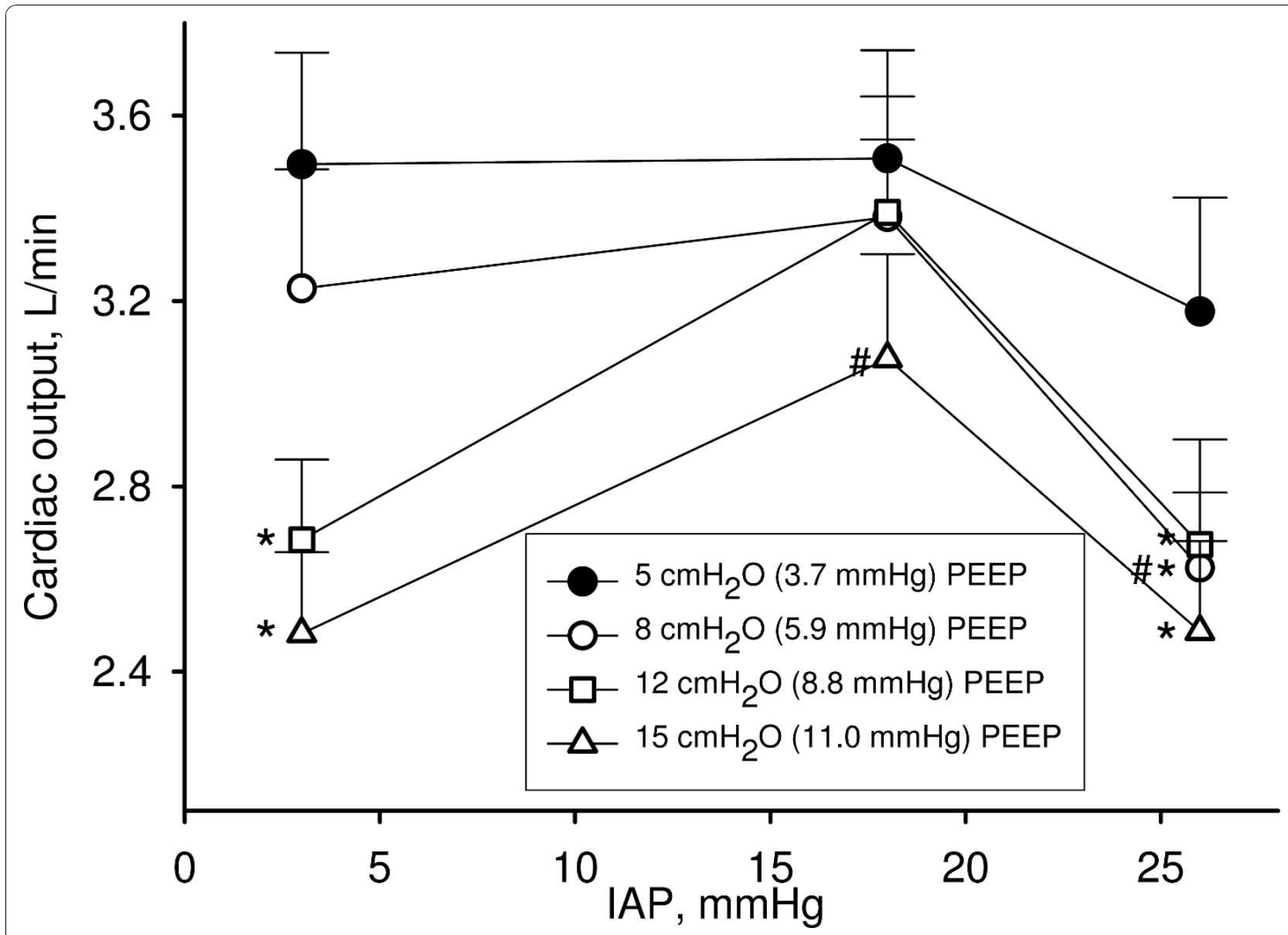

Figure 2 Influence of intra-abdominal pressure and positive end-expiratory pressure on cardiac output. Cardiac output in L/minute in function of different levels of intra-abdominal pressures (IAP) (3 mmHg (baseline), $18 \mathrm{mmHg}$ (grade II intra-abdominal hypertension), and 26 $\mathrm{mmHg}$ (grade IV intra-abdominal hypertension)) at different levels of positive end-expiratory pressures (PEEP). Mean and SE are shown. ANOVA and post hoc Student-Newman-Keuls were used for statistical testing ${ }^{*}, P<0.05$ within an IAP setting vs. the corresponding value at $5 \mathrm{cmH}_{2} \mathrm{O}$ PEEP. \#, $P<0.05$ within a PEEP setting vs. the corresponding value at baseline IAP. For clarification additional symbol is added where necessary.

volume and $\mathrm{CO}$ and $\mathrm{DO}_{2}$ (Tables 1,2 and 3, Figures 1 and 2) which can be attributed to a reduction in venous return [29].

The effect of IAH on $\mathrm{CO}$ is controversial with some studies showing a decrease in $\mathrm{CO}$, while other studies do not show a change or even an increase in $\mathrm{CO}$ in the presence of IAH $[7,10-12,31]$. This controversy can be explained by IAH having a biphasic and potentially opposing effect on $\mathrm{CO}$ which itself may be explained by the dependence of venous return on the level of IAP $[7,10,31]$. Low levels of IAP have been shown to increase venous return as a result of a redistribution of abdominal blood to the thoracic compartment, thus increasing stroke volume and CO [10,31]. However, further increase in IAP overcomes the compensatory effect of blood redistribution from the abdominal compartment to the thoracic compartment decreasing venous return and therefore stroke volume and CO [10,31]. In our study,
IAH did not significantly reduce stroke volume, $\mathrm{CO}$ and $\mathrm{DO}_{2}$ when low levels of PEEP were applied $\left(5 \mathrm{cmH}_{2} \mathrm{O}\right.$, $3.7 \mathrm{mmHg})$. In agreement with other studies [7,11,12], we also found that SVR increased with rising IAP, which may be associated with a reduction in $\mathrm{CO}$ and $\mathrm{DO}_{2}$.

We found that even modest levels of PEEP depressed $\mathrm{CO}$ to a greater extent than IAH alone. This finding is supported by greater depression in $\mathrm{SvO}_{2}$ with PEEP, than with IAP (Figure 4). These findings suggest that PEEP may be detrimental by reducing $\mathrm{DO}_{2}$ and failing to recruit atelectatic lung. If increased levels of PEEP are indicated in the clinical setting, it might be prudent to assess $\mathrm{CO}$ and arterial oxygen saturation before and after increasing the level of PEEP in order to ascertain that the beneficial effect of PEEP with increasing FRC and oxygenation is not offset by a detrimental effect on $\mathrm{CO}$, with a subsequent decrease in $\mathrm{DO}_{2}$. 


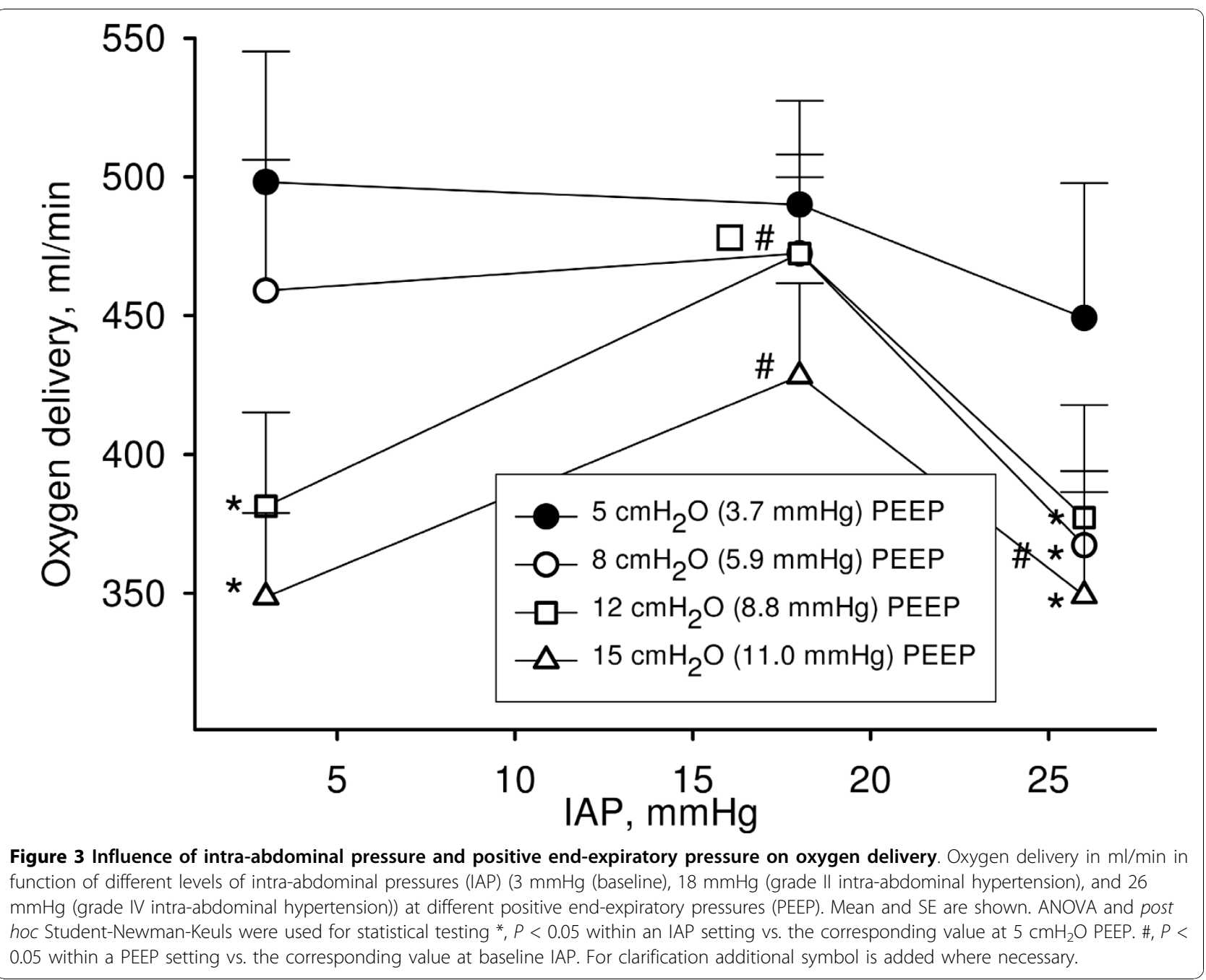

However, since we used healthy lungs in our pig model, the arterial oxygen saturation was nearly $100 \%$ at all IAP and PEEP settings. Therefore, as $\mathrm{DO}_{2}$ is derived from arterial oxygen saturation, haemoglobin levels, and $\mathrm{CO}$, the effect of PEEP and IAP on $\mathrm{DO}_{2}$ paralleled the effect observed on $\mathrm{CO}$ (Figures 2 and 3). It is important to appreciate that our findings cannot be extrapolated to patients with a failing heart, where preload and afterload are more important limitations on $\mathrm{CO}$, or to patients with diseased lungs.

Grade II IAH blunted the effect of PEEP on stroke volume, $\mathrm{CO}$ and $\mathrm{DO}_{2}$. This was possibly caused by an increase in venous return associated with low levels of IAH as outlined above. Grade IV IAH did not protect against the PEEP-induced reduction in stroke volume and $\mathrm{CO}$, most likely due to a reduced venous return associated with high levels of IAH [10,31]. This suggests the existence of IAP levels that are relatively resistant to PEEP induced $\mathrm{CO}$ reduction by counteracting the reduction in venous return caused by increasing levels of PEEP.

\section{Influence of PEEP on IAP}

PEEP up to $15 \mathrm{cmH}_{2} \mathrm{O}(11.0 \mathrm{mmHg})$ did not further increase IAP. Other investigators have found either absent or minimal influence of PEEP on IAP and it appears that an effect of PEEP on IAP can only be expected when PEEP approximates IAP [32-35]. Therefore our findings that PEEP did not influence IAP can be attributed to the relatively modest level of PEEP $\left(15 \mathrm{cmH}_{2} \mathrm{O}, 11 \mathrm{mmHg}\right)$ in comparison to the levels of IAP $(18 \mathrm{mmHg}$ and 26 $\mathrm{mmHg}$ ) used in this study (estimated trans-pulmonary PEEP of -7 and $-15 \mathrm{mmHg}$, respectively).

\section{Limitations}

We used pigs in this study because pig models have been used extensively in IAH research and the physiology of this animal is very similar to humans. 


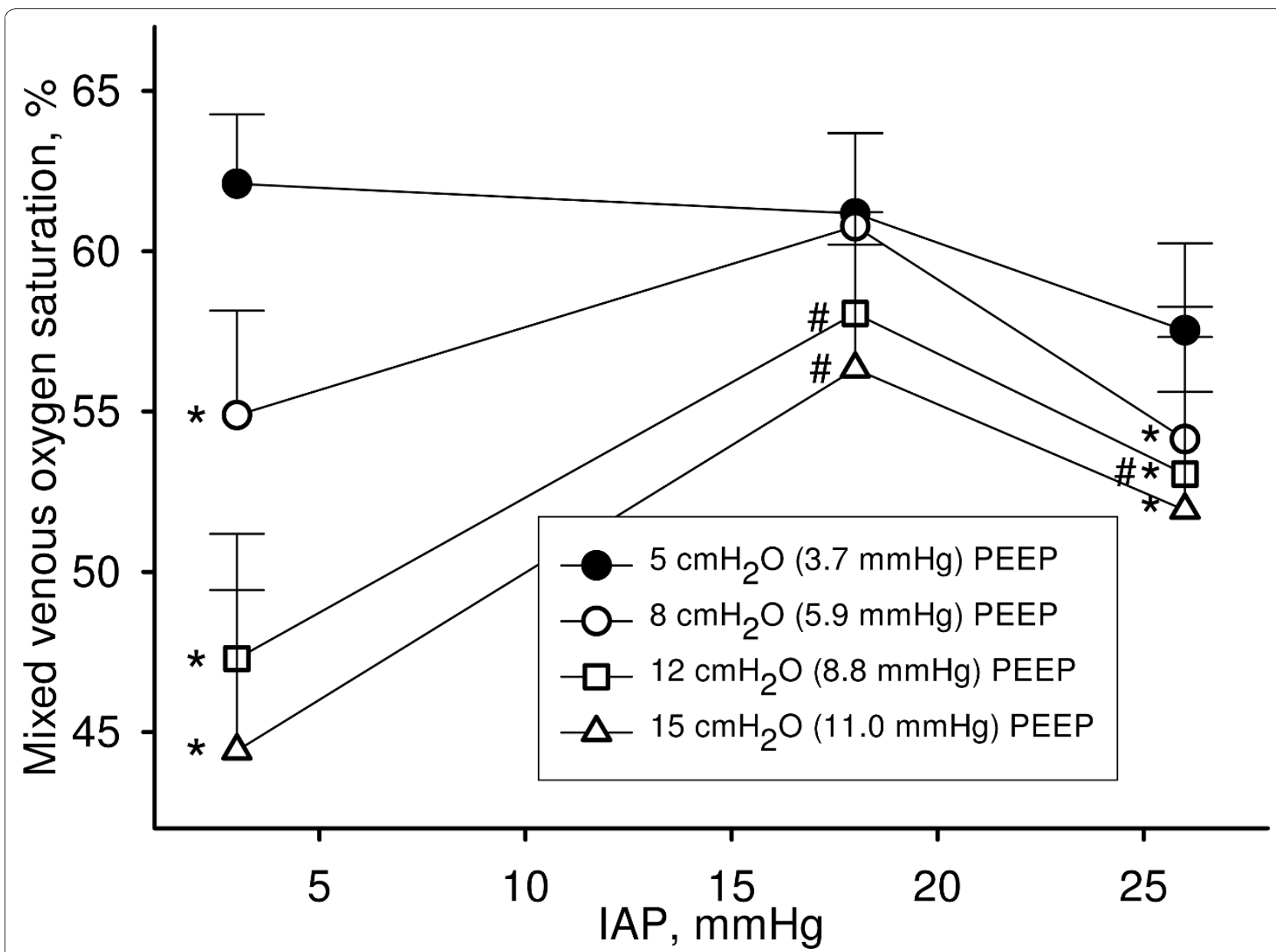

Figure 4 Influence of intra-abdominal pressure and positive end-expiratory pressure on mixed venous oxygen saturation. Mixed venous oxygen saturation in \% in function of different levels of intra-abdominal pressures (IAP) (3 mmHg (baseline), $18 \mathrm{mmHg}$ (grade II intraabdominal hypertension), and $26 \mathrm{mmHg}$ (grade IV intra-abdominal hypertension)) at different levels of positive end-expiratory pressures (PEEP). Mean and SE are shown. ANOVA and post hoc Student-Newman-Keuls were used for statistical testing $*, P<0.05$ within an IAP setting vs. the corresponding value at $5 \mathrm{cmH}_{2} \mathrm{O}$ PEEP. \#, $P<0.05$ within a PEEP setting vs. the corresponding value at baseline IAP.

However, it is always difficult to transfer animal data into clinical practice, especially when applying higher levels of PEEP in healthy pigs with IAH. Therefore, an extrapolation of our results onto the effects of IAP and PEEP in critically ill patients remains difficult.

We used an inflatable balloon to achieve different levels of IAP as a model of acute IAH [19]. We chose not to use a pneumoperitoneum using gas inflation as used by some other investigators for two reasons. First, we wanted to eliminate the cardiovascular and respiratory response to hypercapnia when carbon dioxide or air is used when performing pneumoperitoneum [12]. Second, we wanted to measure the influence of PEEP on IAP and this is difficult to perform in the setting of a pneumoperitoneum due to possible gas leakage.

Ideally, in order to imitate the clinical setting as closely as possible, a fluid based IAH model should be used (haemorrhage, ascites, oedema). However, models using fluid instillation have their own disadvantages mainly due to uncontrollable abdominal fluid absorption with possible change in cardio-respiratory physiology [36,37].

To ensure the absence of changes in IAP caused by leakage from the balloon, we assessed the changes in IAP over time. As there were no significant changes in IAP before and after the five-minute stabilization period, we conclude that there was insignificant gas leakage from the intra-abdominal balloon or adaptive abdominal processes.

As we used healthy pigs in our experimental model it is not surprising that we obtained high $\mathrm{PaO}_{2}$ levels and a near $100 \%$ arterial oxygen saturation at all IAP and PEEP settings. We used a porcine mathematical model to calculate oxygen saturation that shows a good agreement with the measured oxygen saturation [23].

As we did not use an oesophageal catheter to measure pleural pressures, we are unable to give information on 
chest wall compliance, which is strongly influenced by IAP in the setting of IAH [33]. Trans-pulmonary pressures have been shown to be useful in titrating the level of PEEP in the setting of acute respiratory distress syndrome [38]. In the setting of IAH, trans-pulmonary pressures have been recommended not only to help titrate the level of PEEP but also to guide recruitment manoeuvres [13]. As we limited our recruitment manoeuvres to a maximum of $40 \mathrm{cmH}_{2} \mathrm{O}$ airway pressure and not to maximum trans-pulmonary pressures of $25 \mathrm{cmH}_{2} \mathrm{O}$ we were not able to perform sufficient recruitment in all PEEP and IAP settings, especially at $26 \mathrm{mmHg}$ of IAP. This might explain the absent effect of PEEP in reversing IAP induced FRC decline in the setting of grade IV IAH, respectively. However, we think this reduced influence of PEEP in reversing IAP induced FRC decline is better explained by the relative small estimated trans-pulmonary PEEP $(-7 \mathrm{mmHg}$ and $-15 \mathrm{mmHg}$ at PEEP of $11 \mathrm{mmHg}$ and IAP of 18 and $26 \mathrm{mmHg}$, respectively).

We chose four PEEP settings and three IAP settings in our experimental model, as our main focus was to study the effect of PEEP on FRC, $\mathrm{CO}$ and $\mathrm{DO}_{2}$ in the setting of increased IAP. We used PEEP values of $5,8,12$, and $15 \mathrm{cmH}_{2} \mathrm{O}$ as these PEEP values are frequently applied ventilator settings in critically ill patients. Since it remains unclear what the exact threshold value of IAP is at which a surgical abdominal decompression should be performed, we chose grade II and grade IV IAH because surgical abdominal decompression is currently not recommended for grade II whereas it is recommended for persistent grade III and IV in the presence of a new organ failure [1,2].

Another limitation is that we measured the mean IAP instead of the end-expiratory IAP as suggested by the World Society of Abdominal Compartment Syndrome [1]. As it has been shown that the difference between end-inspiratory and end-expiratory IAP increases in proportion to IAP, our measured mean IAP will underestimate end-expiratory IAP by approximately $1 \mathrm{mmHg}$ at $11 \mathrm{mmHg}$ end-expiratory IAP [39].

\section{Conclusions}

The results of this experimental study show that IAH had only a minimal effect on $\mathrm{CO}$ and $\mathrm{DO}_{2}$ whereas FRC was markedly and $\mathrm{PaO}_{2}$ levels were minimally reduced with increasing levels of IAH. On the other hand, commonly applied PEEP levels of up to $15 \mathrm{cmH}_{2} \mathrm{O}(11.0$ $\mathrm{mmHg}$ ) only partially restored FRC in grade II IAH and had no effect in grade IV IAH. At the same time increasing levels of PEEP may have a detrimental effect on $\mathrm{CO}$ and $\mathrm{DO}_{2}$ at high levels of IAH.

Based on these results, prophylactic PEEP levels inferior to the corresponding IAP can not be recommended in the setting of IAH as these PEEP levels are not sufficient in preventing FRC decline caused by IAH and may even be associated with a reduced $\mathrm{DO}_{2}$ as a consequence of a decreased CO. Further trials to assess whether higher levels of PEEP can reverse IAP induced FRC decline without impairing $\mathrm{CO}$ in the setting of IAH are required in the future.

\section{Key messages}

- In this pig model, the application of commonly applied levels of PEEP (up to $15 \mathrm{cmH}_{2} \mathrm{O}$ ) was not able to prevent a FRC decline caused by IAH $(18 \mathrm{mmHg}$ and $26 \mathrm{mmHg}$ ).

- CO decreased with increasing levels of PEEP but not with increasing levels of IAH.

- Based on these results, prophylactic PEEP levels inferior to the corresponding IAP can not be recommended in the setting of IAH as these PEEP levels are not sufficient in preventing the FRC decline caused by IAH and may be associated with a reduced $\mathrm{CO}$.

- Increasing the level of PEEP from 5 to $15 \mathrm{cmH}_{2} \mathrm{O}$ did not further increase IAP in the setting of IAH.

\section{Abbreviations}

APP: abdominal perfusion pressure; Cdyn: dynamic compliance; CO: cardiac output; $\mathrm{DO}_{2}$ : oxygen delivery; $\mathrm{FRC}$ : functional residual capacity; $\mathrm{Hb}$ : haemoglobin concentration; IAH: intra-abdominal hypertension; IAP: intraabdominal pressure; IV: intravenous; MAP: mean arterial blood pressure; mPaw: mean airway pressure; $\mathrm{PaO}_{2}$ : arterial oxygen tension; PEEP: positive end-expiratory pressure; pPaw: peak airway pressure; SVR: systemic vascular resistance.

\section{Acknowledgements}

This study was supported by the Sir Charles Gairdner Hospital Research Fund, by the Sir Charles Gairdner Hospital Intensive Care Research Fund. We thank Richard Parsons for statistical support. We thank the Department of Medical Technology and Physics as well as the team of the Large Animal Facility of the University of Western Australia for technical assistance.

\section{Author details}

${ }^{1}$ Intensive Care Unit, Sir Charles Gairdner Hospital, Hospital Avenue, Nedlands (Perth) WA 6009, Australia. ${ }^{2}$ Veterinary Anaesthesia, Murdoch University Veterinary Hospital, 90 South Street, Murdoch (Perth) WA 6150, Australia. ${ }^{3}$ Department of Pulmonary Physiology, Sir Charles Gairdner Hospital, Hospital Avenue, Nedlands (Perth) WA 6009, Australia.

\section{Authors' contributions}

$A R, L H, G M, B S$ and PVH participated in the design of the study. AR, LH, GM, $\mathrm{BR}$ and $\mathrm{BN}$ contributed to data collection. AR performed the statistical analyses and drafted the manuscript. $L H, G M, B R, B S$ and $P V H$ revised the manuscript. All authors read and approved the final manuscript.

\section{Competing interests}

The authors declare that they have no competing interests.

Received: 5 January 2010 Revised: 28 April 2010 Accepted: 2 July 2010 Published: 2 July 2010

\section{References}

1. Malbrain ML, Cheatham ML, Kirkpatrick A, Sugrue M, Parr M, De Waele J, Balogh Z, Leppäniemi A, Olvera C, Ivatury R, D'Amours S, Wendon J, 
Hillman K, Johansson K, Kolkman K, Wilmer A: Results from the International Conference of Experts on Intra-abdominal Hypertension and Abdominal Compartment Syndrome. I. Definitions. Intensive Care Med 2006, 32:1722-1732.

2. Cheatham ML, ML Kirkpatrick A, Sugrue M, Parr M, De Waele J, Balogh Z, Leppäniemi A, Olvera C, Ivatury R, D'Amours S, Wendon J, Hillman K, Wilmer A: Results from the International Conference of Experts on Intraabdominal Hypertension and Abdominal Compartment Syndrome. II. Recommendations. Intensive Care Med 2007, 33:951-962.

3. Malbrain ML, Chiumello D, Pelosi P, Wilmer A, Brienza N, Malcangi V, Bihari D, Innes R, Cohen J, Singer P, Japiassu A, Kurtop E, De Keulenaer BL, Daelemans R, Del Turco M, Cosimini P, Ranieri M, Jacquet L, Laterre PF, Gattinoni L: Prevalence of intra-abdominal hypertension in critically ill patients: a multicentre epidemiological study. Intensive Care Med 2004 30:822-829.

4. Malbrain ML, Chiumello D, Pelosi P, Bihari D, Innes R, Ranieri VM, Del Turco M, Wilmer A, Brienza N, Malcangi V, Cohen J, Japiassu A, De Keulenaer BL, Daelemans R, Jacquet L, Laterre PF, Frank G, de Souza P, Cesana B, Gattinoni L: Incidence and prognosis of intraabdominal hypertension in a mixed population of critically ill patients: a multiplecenter epidemiological study. Crit Care Med 2005, 33:315-322.

5. Vidal MG, Ruiz Weisser J, Gonzalez F, Toro MA, Loudet C, Balasini C, Canales $H$, Reina R, Estenssoro E: Incidence and clinical effects of intraabdominal hypertension in critically ill patients. Crit Care Med 2008, 36:1823-1831.

6. Reintam A, Parm P, Kitus R, Kern H, Starkopf J: Primary and secondary intra-abdominal hypertension-different impact on ICU outcome. Intensive Care Med 2008, 34:1624-1631.

7. Cheatham ML, Malbrain ML: Cardiovascular implications of abdominal compartment syndrome. Acta Clin Belg Suppl 2007, 98-112

8. Cheatham ML, White MW, Sagraves SG, Johnson JL, Block EF: Abdominal perfusion pressure: a superior parameter in the assessment of intraabdominal hypertension. J Trauma 2000, 49:621-626, discussion 626-627.

9. Doty JM, Oda J, Ivatury RR, Blocher CR, Christie GE, Yelon JA, Sugerman HJ: The effects of haemodynamic shock and increased intra-abdominal pressure on bacterial translocation. J Trauma 2002, 52:13-17.

10. Kitano Y, Takata M, Sasaki N, Zhang Q, Yamamoto S, Miyasaka K: Influence of increased abdominal pressure on steady-state cardiac performance. J Appl Physiol 1999, 86:1651-1656

11. Vivier E, Metton O, Piriou V, Lhuillier F, Cottet-Emard JM, Branche P, Duperret $S$, Viale JP: Effects of increased intra-abdominal pressure on central circulation. Br J Anaesth 2006, 96:701-707.

12. Sharma KC, Brandstetter RD, Brensilver JM, Jung LD: Cardiopulmonary physiology and pathophysiology as a consequence of laparoscopic surgery. Chest 1996, 110:810-815.

13. Pelosi $P$, Quintel M, Malbrain ML: Effect of intra-abdominal pressure on respiratory mechanics. Acta Clin Belg Supp/ 2007, 78-88.

14. Suwanvanichkij V, Curtis JR: The use of high positive end-expiratory pressure for respiratory failure in abdominal compartment syndrome. Respir Care 2004, 49:286-290.

15. Sugrue M, D'Amours S: The problems with positive end expiratory pressure (PEEP) in association with abdominal compartment syndrome (ACS). J Trauma 2001, 51:419-420.

16. Kallet RH, Siobal MS, Alonso JA, Warnecke EL, Katz JA, Marks JD: Lung collapse during low tidal volume ventilation in acute respiratory distress syndrome. Respir Care 2001, 46:49-52.

17. von Ungern-Sternberg BS, Regli A, Schibler A, Hammer J, Frei FJ, Erb TO: The impact of positive end-expiratory pressure on functional residual capacity and ventilation homogeneity impairment in anesthetized children exposed to high levels of inspired oxygen. Anesth Analg 2007, 104:1364-1368.

18. Malbrain ML: Abdominal pressure in the critically ill: measurement and clinical relevance. Intensive Care Med 1999, 25:1453-1458.

19. Engum SA, Kogon B, Jensen E, Isch J, Balanoff C, Grosfeld JL: Gastric tonometry and direct intraabdominal pressure monitoring in abdominal compartment syndrome. J Pediatr Surg 2002, 37:214-218.

20. EDGAR, randomisation for two treatment factors; split plots. [http://www. edgarweb.org.uk/choosedesign.htm].

21. Tusman G, Bohm SH, Vazquez de Anda GF, Tusman G, Bohm SH, Vazquez de Anda GF: 'Alveolar recruitment strategy' improves arterial oxygenation during general anaesthesia. Br J Anaesth 1999, 82:8-13.
22. Morris MG, Gustafsson P, Tepper R, Gappa M, Stocks J, ERS/ATS Task Force on Standards for Infant Respiratory Function Testing: The bias flow nitrogen washout technique for measuring the functional residual capacity in infants. ERS/ATS Task Force on Standards for Infant Respiratory Function Testing. Eur Respir J 2001, 17:529-536.

23. Serianni R, Barash J, Bentley T, Sharma P, Fontana JL, Via D, Duhm J, Bunger R, Mongan PD: Porcine-specific hemoglobin saturation measurements. J Appl Physiol 2003, 94:561-566.

24. Lumb AB: Nunn's Applied Respiratory Physiology. Philadelphia: Butterworth-Heinemann, 62005.

25. Cheung PY, Abozaid S, Al-Salam Z, Johnson S, Li Y, Bigam D: Systemic and regional haemodynamic effects of high-dose epinephrine infusion in hypoxic piglets resuscitated with 100\% oxygen. Shock 2007, 28:491-497.

26. Quintel $M$, Pelosi $P$, Caironi $P$, Meinhardt JP, Luecke T, Herrmann $P$, Taccone P, Rylander C, Valenza F, Carlesso E, Gattinoni L: An increase of abdominal pressure increases pulmonary edema in oleic acid-induced lung injury. Am J Respir Crit Care Med 2004, 169:534-541.

27. Krebs J, Pelosi P, Tsagogiorgas C, Alb M, Luecke T: Effects of positive endexpiratory pressure on respiratory function and haemodynamics in patients with acute respiratory failure with and without intra-abdominal hypertension: a pilot study. Crit Care 2009, 13:R160.

28. Valenza F, Chevallard G, Porro GA, Gattinoni L: Static and dynamic components of esophageal and central venous pressure during intraabdominal hypertension. Crit Care Med 2007, 35:1575-1581.

29. Putensen $C$, Wrigge $H$, Hering $R$ : The effects of mechanical ventilation on the gut and abdomen. Curr Opin Crit Care 2006, 12:160-165.

30. Suter PM, Fairley B, Isenberg MD: Optimum end-expiratory airway pressure in patients with acute pulmonary failure. N Engl J Med 1975, 292:284-289.

31. Takata M, Wise RA, Robotham JL: Effects of abdominal pressure on venous return: abdominal vascular zone conditions. J Appl Physiol 1990, 69:1961-1972.

32. Ferrer C, Piacentini EA, Molina E, Trenado J, Sanchez B, Nava JM: Higher PEEP levels results in small increases in intra-abdominal pressure in critical care patients. Intensive Care Med 2008, 34:5140

33. Gattinoni L, Pelosi P, Suter PM, Pedoto A, Vercesi P, Lissoni A: Acute respiratory distress syndrome caused by pulmonary and extrapulmonary disease. Different syndromes? Am J Respir Crit Care Med 1998, 158:3-11.

34. Sussman AM, Boyd CR, Williams JS, DiBenedetto RJ: Effect of positive endexpiratory pressure on intra-abdominal pressure. South Med J 1991, 84:697-700.

35. De Keulenaer BL, De Waele JJ, Powell B, Malbrain ML: What is normal intra-abdominal pressure and how is it affected by positioning, body mass and positive end-expiratory pressure? Intensive Care Med 2009, 35:969-976.

36. Meier C, Contaldo C, Schramm R, Holstein JH, Hamacher J, Amon M, Wanner G, Trentz O, Menger MD: A new model for the study of the abdominal compartment syndrome in rats. J Surg Res 2007, 139:209-216.

37. Schachtrupp A, Wauters J, Wilmer A: What is the best animal model for ACS? Acta Clin Belg Suppl 2007, 225-232.

38. Talmor D, Sarge T, Malhotra A, O'Donnell CR, Ritz R, Lisbon A, Novack V, Loring SH: Mechanical ventilation guided by esophageal pressure in acute lung injury. N Engl J Med 2008, 359:2095-2104

39. Sturini E, Saporito A, Sugrue M, Parr MJ, Bishop G, Braschi A: Respiratory variation of intra-abdominal pressure: indirect indicator of abdominal compliance? Intensive Care Med 2008, 34:1632-1637.

\section{doi:10.1186/cc9095}

Cite this article as: Regli et al:: Commonly applied positive endexpiratory pressures do not prevent functional residual capacity decline in the setting of intra-abdominal hypertension: a pig model. Critical Care 2010 14:R128. 